\title{
Comparative Prospective Evaluation of the Effectiveness of Long- pulsed Nd-YAG Laser and Fractional Er-YAG Laser Treatments in Keloid and Hypertrophic Scars
}

\author{
(D) Ayșe Mine Gök, (1) Özge Așkın, (1) Zekayi Kutlubay
}

Istanbul University-Cerrahpasa, Cerrahpasa Faculty of Medicine, Department of Dermatology and Venereology, Istanbul, Turkey

\section{ABSTRACT}

Background: Keloid and hypertrophic scars are pathological scars that occur as a result of the abnormal healing process of the skin against trauma. It bothers patients cosmetically and causes itching, pain, burning, sensitivity and loss of function in patients. There is no single treatment with proven efficacy in the treatment of keloid and hypertrophic scars. Laser treatments, a relatively new modality, are increasingly being used in keloid and hypertrophic scars treatment to reduce the risk of relapse and achieve positive cosmetic and symptomatic results. In our study, we aimed to compare the effectiveness of long-pulsed Nd-YAG laser and fractional Er-YAG laser treatments in the treatment of keloid and hypertrophic scars.

Materials and Methods: Twenty patients with keloid and/or hypertrophic scars were included in the study. Patients with keloid and/or hypertrophic scars were divided into two groups of ten, each for five sessions of laser treatment. Group A received fractional Er: YAG laser, group B received long-pulsed Nd: YAG laser treatment. Patients were evaluated before treatment and their lesions were photographed. The patients were evaluated with the Stony Brook Scar Evaluation Scale and the treatment satisfaction scale three months after the treatment was completed.

Results: There was no change in Stony Brook Scar Evaluation Scale scores before and after treatment in both groups. After the treatment, eight patients were satisfied with the treatment, and twelve patients were not satisfied with the treatment.

Conclusion: Long-pulsed Nd-YAG laser and fractional Er-YAG laser treatments have not been shown to be effective in the treatment of keloid and/or hypertrophic scars.

Keywords: Keloid, Hypertrophic scars, Long-pulsed Nd-YAG laser, Fractional Er-YAG laser

\section{Introduction}

Keloid and hypertrophic scar are forms of pathological healing resulting from an abnormal response of the skin to trauma [1]. These wound healing disorders both cosmetically disturb patients and cause itching, pain, burning, sensitivity and loss of function in patients [2]. Quality of life is affected by functional impairment and psychosocial burden in affected individuals [3].
Keloids extend into normal skin beyond the original lesion confine and cannot be stretched. However, hypertrophic scars remain within the confines of the original lesion, but may increase or decrease in size over months or years [4].

The pathogenesis of keloid and hypertrophic scar is not fully known. Changes in the wound healing process and many local and genetic factors are thought to play a role in the pathogenesis $[5,6,7]$. 
The methods used in the treatment of keloid and hypertrophic scars are intralesional or topical corticosteroids, cryotherapy, radiotherapy, silicone gel, compression, topical imiquimod, other intralesional treatments (5-FU, bleomycin, interferon), surgical repairs and laser treatments. Patients' response to these treatments is variable [8]. Because of the painful use of intralesional steroids and cytostatic agents, side effects such as atrophy and telangiectasia, hypopigmentation after cryotherapy, pigmentation disorders and ulceration after radiotherapy, physicians have been pushed to investigate new treatment methods. Recent studies show that laser applications can be an effective and safe method in the treatment of keloids and hypertrophic scars.

Fractional ablative lasers promote wound healing and cause textural remodeling of scars. Nd: YAG laser is preferred in keloid and hypertrophic scar treatment due to its ability to heat both collagen and vascular elements in the dermis [9].

\section{Materials and Methods}

Twenty patients who were admitted to outpatient clinic of the Dermatology Department, Istanbul University-Cerrahpaşa Cerrahpaşa Medical Faculty due to keloid and/or hypertrophic scar were included in this prospective study. Patients under the age of 18; immunocompromised, pregnant or breastfeeding; with an acute bacterial or viral infection on the lesion; with a history of skin cancer; and those that are sensitive to light were excluded from this study. In the pre-treatment evaluation, each patient's age, gender, Fitzpatrick skin type, previous treatments, number of lesions, lesion localization, lesion formation pattern and Stony Brook Scar Evaluation Scale scores were noted. Clinical photographs of the lesions of each patient were taken before the treatment.

Patients with keloid and/or hypertrophic scars were divided into two groups of ten each. Group A received fractional Er: YAG laser and group B long-pulsed Nd: YAG laser treatment.

Ten patients in group A received a total of five sessions of fractional Er: YAG laser (Fotona; Dualis SP, Ljubljana, Slovenia) at 4-6 week intervals. The laser parameters were a fluency of $8.4 \mathrm{~J}$, a $3 \mathrm{~mm}$ spot size and a frequency of $3 \mathrm{~Hz}$. All lesions of the patient were treated with the specified parameters with two passes.

Ten patients in group $B$ received a total of five sessions of longpulsed Nd: YAG laser (Fotona; Dualis SP, Ljubljana, Slovenia) at 4-6 week intervals. The laser parameters were a fluency of $180 \mathrm{~J}$, a spot size of $2 \mathrm{~mm}$, and a frequency of $3 \mathrm{~Hz}$. All lesions of the patient were treated with the specified parameters with two passes.

All laser treatments were performed after applying 2\% lidocaine topically under occlusion for 30 minutes. No other wound treatments were performed between laser treatment sessions to avoid bias in our results. Patients were evaluated at each session for adverse effects including pain, erythema, infection, and hyperpigmentation or hypopigmentation.

All lesions of each patient were evaluated using the Stony Brook Scar Evaluation Scale three months after the last laser treatment, and each lesion was photographed. In addition, each patient was evaluated with a 4-point satisfaction scale three months after the last laser session.

The approval of Istanbul University-Cerrahpasa, Cerrahpasa Medical Faculty Ethics Committee was taken before initiating the study. The informed consent of each patient was taken.

\section{Results}

Of the twenty patients included in the study, six were male and fourteen were female. The mean age of the patients was 29.8 years; with the youngest being 18 years old and the oldest being 54 years old. The Fitzpatrick skin types of the patients ranged from 2 to 3. The most commonly used treatments before laser treatment were intralesional corticosteroid injections and cryotherapy. Twenty patients had 141 lesions in total. The lesions were most frequently located in the shoulders and the second most frequently in the chest. Keloid and/or hypertrophic scar formation developed after trauma in one patient, after surgery in seven patients, after burn in two patients, and after acne vulgaris in four patients.

Fractional Er: YAG laser treatment was applied to all lesions of ten patients in group A. The average age of patients in group A was 24.4 years. Six of the patients in this group were women and four were men, and the total number of lesions was ninety-three. Lesions most frequently developed after acne vulgaris in patients in this group. Stony Brook Scar Evaluation Scale score was found to be 1 for each of the ninety-three lesions before treatment. There was no change in the Stony Brook Scar Evaluation Scale scores of the lesions that were re-evaluated 3 months after five sessions of laser treatment. Most of the patients were not satisfied with the treatment in the satisfaction assessment scale performed three months after the end of the treatment. Three patients stated that their itching complaints decreased after the treatment. All ten patients who received treatment had post-treatment burning and reduced redness within a few days. None of the patients had side effects such as postlesional hyperpigmentation or postlesional hypopigmentation. Figure 1a shows the pre-treatment of fractional Er: YAG laser treatment of a 25-year-old female patient who developed a keloid after acne on the anterior chest; and Figure $1 \mathrm{~b}$ shows 3 months after the $5^{\text {th }}$ treatment session of the same patient. Nd: YAG laser treatment was applied to all lesions of ten patients in group B. The average age of patients in group B was 35.5 years. Eight of the patients in this group were women and two were men, and the total number of lesions was forty-eight. Lesions most frequently developed after 
surgery in patients in this group. Stony Brook Scar Evaluation Scale score was found to be zero in three lesions before treatment and one in other lesions. There was no change in the Stony Brook Scar Evaluation Scale scores of the lesions that were re-evaluated three months after five sessions of laser treatment. Most of the patients were not satisfied with the treatment in the satisfaction assessment scale performed three months after the end of the treatment. Four patients stated that their itching complaints decreased after the treatment. Pain complaints during treatment were more common in patients compared to the other group. All ten patients who received treatment had post-treatment burning and reduced redness within a few days. None of the patients had side effects such as postlesional hyperpigmentation or postlesional hypopigmentation. Figure 2a shows the pre-long-pulsed Nd: YAG laser treatment of a 54-year-old male patient with a keloid developed at the suture line after surgery under the left breast; and Figure $2 \mathrm{~b}$ shows 3 months after the $5^{\text {th }}$ treatment session of the same patient.
The results revealed that neither of the laser treatments had any effect on hypertrophic scar and/or keloid treatment.

\section{Discussion}

This study investigated and compared the effectiveness of fractional Er: YAG laser and long-pulsed Nd: YAG lasers for treating keloid and hypertrophic scars.

In the study published by Koike et al. [10] in 2014, the effectiveness of long-pulsed Nd: YAG laser was evaluated in 102 patients. The patients were treated for a year with the treatment applied every 3-4 weeks. The scars were evaluated using the Japan Scar Workshop Scar Scale 2011 before starting treatment and one month after the last session. As a result of the study, it was observed that there was a significant decrease in the Japan Scar Workshop score in keloid and hypertrophic scars.

In the study published by Al-Mohamady et al. [11] in 2016, the effectiveness of pulsed dye laser and long-pulsed $\mathrm{Nd}$ : YAG laser

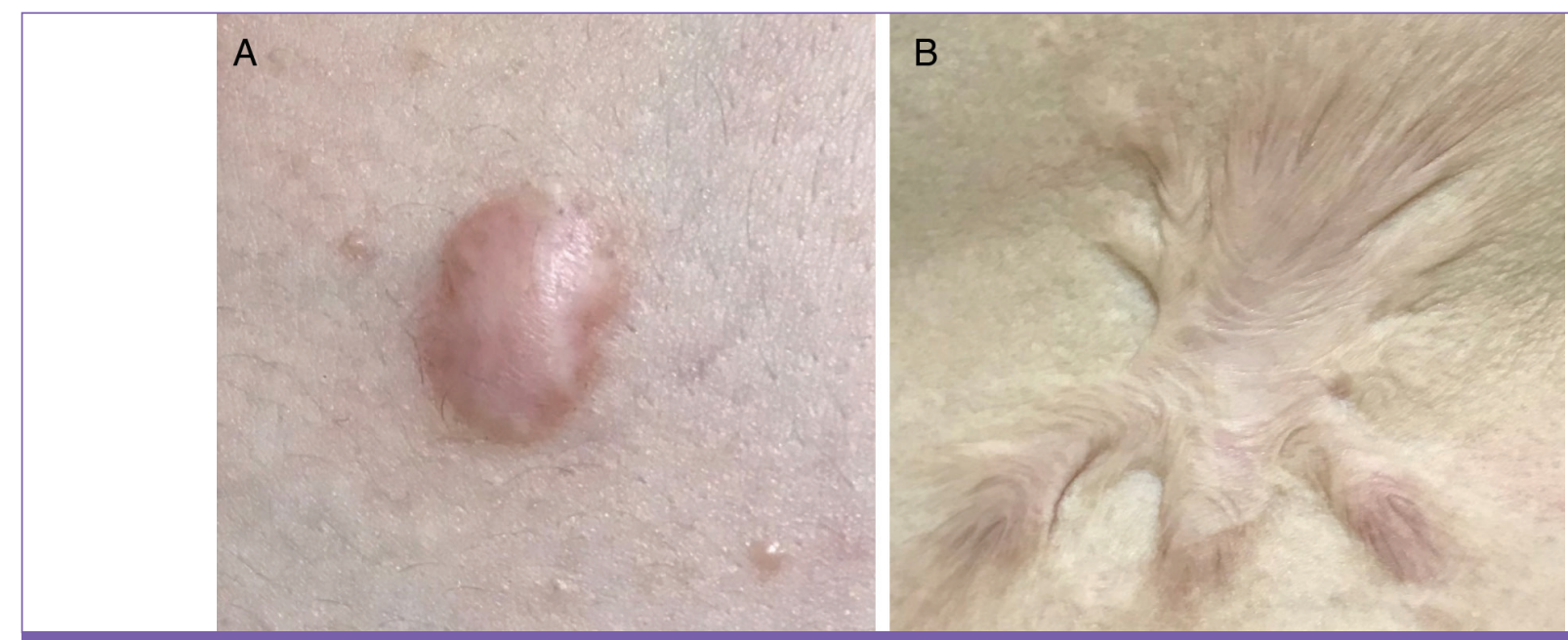

Figure 1. A) Pre-treatment of two patients treated with fractional Er: YAG laser. B) Three months after the $5^{\text {th }}$ treatment session of two patients treated with fractional Er: YAG laser
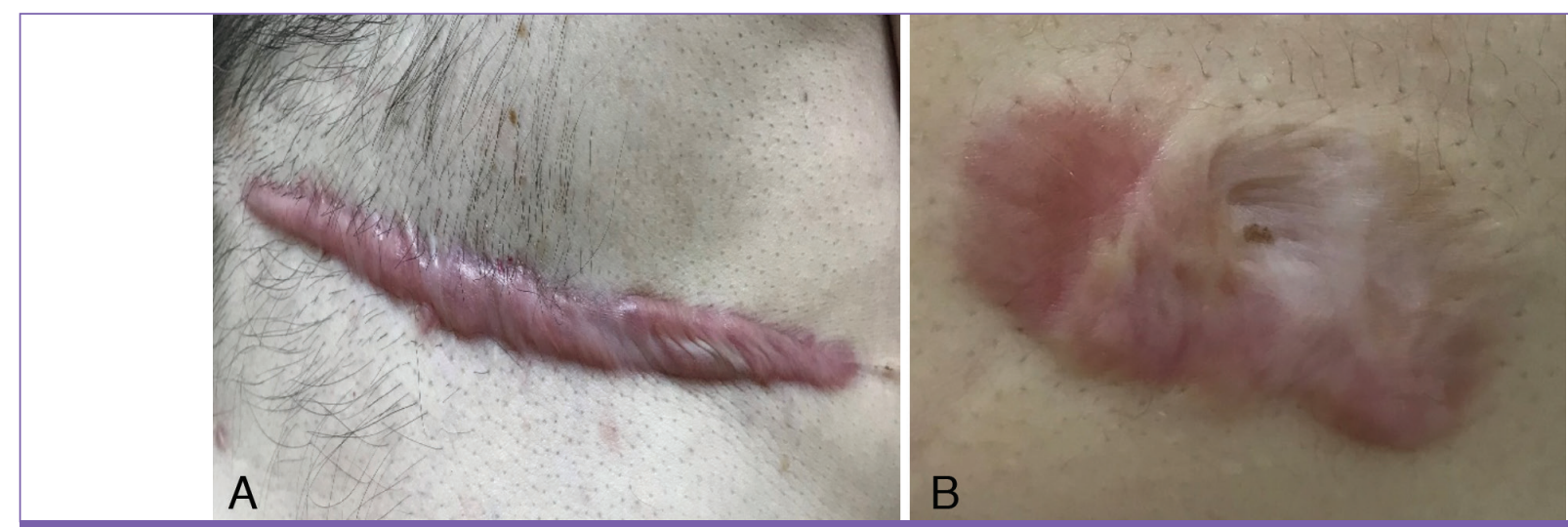

Figure 2. A) Pre-treatment of two patients treated with long-pulsed Nd: YAG laser. B) Three months after the $5^{\text {th }}$ treatment session of two patients treated with long-pulsed Nd: YAG laser 
was compared in a study involving twenty patients with keloid and hypertrophic scar. Pulsed dye laser was applied to half of the lesions and long-pulsed Nd: YAG laser treatment was applied to the other half six times with one month intervals. Lesions were assessed using the Vancouver scar scale at baseline and one month after the last laser session. While significant improvements were achieved in Vancouver scar scale after treatment with both lasers, no statistically significant difference was found between the pulsed dye laser and long-pulsed Nd: YAG laser treated areas.

The effectiveness of fractional $\mathrm{CO}_{2}$ laser and long-pulsed $\mathrm{Nd}$ : YAG laser in 30 patients with keloid and hypertrophic scar, published in 2020 by Tawfic et al. [9]. Three scars were selected in each patient, and fractional CO2 laser, long-pulsed Nd: YAG laser and a combination of both were applied to the lesions 4 times at 4-6 week intervals. Patients were evaluated before starting treatment and 1 month after the last session using the Vancouver Scar Scale and the Patient and Observer Scar Assessment Scale. At the end of the study, long-pulsed Nd: YAG laser was found to be effective and safe in the treatment of hypertrophic scars and keloids, while fractional $\mathrm{CO}_{2}$ laser provided better healing in hypertrophic scars compared to keloids. In lesions where both lasers were applied in combination, no significant additional benefit was seen and the side effect profile was higher.

In the study published by Choi et al. [12] in 2014, the efficiency of fractional $\mathrm{CO}_{2}$ laser and fractional Er: YAG laser was compared in 23 patients with hypertrophic scar. In the study in which 10 patients were treated with fractional $\mathrm{CO}_{2}$ laser and 13 patients with fractional Er: YAG laser, treatment responses were evaluated using the Vancouver Scar Scale, the evaluation of healing based on photographs and the scale showing the satisfaction of the patients. As a result of the study, fractional $\mathrm{CO}_{2}$ laser was found to be more effective than fractional Er: YAG laser treatment in hypertrophic scar treatment.

In the literature, the mechanism of action of laser treatment in scar treatment is explained as heat generation that initiates inflammation and causes an increase in vascular permeability, matrix metalloproteinase production and collagen fiber fascicle decomposition. This shows that lasers will be one of the alternative treatment methods in the treatment of keloid and hypertrophic scars, which have many treatment options, but with these options, which have high recurrence and side effects. In the literature, there are studies in which long-pulsed Nd: YAG laser and fractional Er: YAG laser are used and found effective in the treatment of keloid and hypertrophic scar. However, there is no study comparing longpulsed ND: YAg laser and fractional Er: YAG laser in the treatment of keloid and hypertrophic scar in the literature. Also, contrary to what is stated in the literature, the benefit of long-pulsed Nd: YAG laser and fractional Er: YAG laser treatment in keloid and hypertrophic scar treatment was not observed in our study.

\section{Study Limitations}

The limitation of our study is that the number of patients is low due to the intervening COVID-19 pandemic.

\section{Conclusion}

Finally, this is the first study comparing the efficacy of long-pulsed $\mathrm{Nd}$ : YAG laser and fractional Er: YAG laser in the treatment of keloid and hypertrophic scars. Again, contrary to what is stated in the literature, successful results have not been obtained in keloid and hypertrophic scar treatment with long-pulsed Nd: YAG laser and fractional Er: YAG laser. Studies with greater sample sizes are needed to further clarify these matters.

\section{Ethics}

Ethics Committee Approval: The study were approved by the Istanbul University-Cerrahpasa, Cerrahpasa Faculty of Medicine of Local Ethics Committee (protocol number: F-01, date: 02.04.2019).

Informed Consent: The informed consent of each patient was taken.

Peer-review: Externally and internally peer-reviewed.

\section{Authorship Contributions}

Surgical and Medical Practices: A.M.G., Ö.A., Concept: A.M.G., Ö.A., Z.K., Design: A.M.G., Ö.A., Z.K., Data Collection or Processing: A.M.G., Ö.A., Analysis or Interpretation: A.M.G., Ö.A., Literature Search: A.M.G., Writing: A.M.G.

Conflict of Interest: No conflict of interest was declared by the authors.

Financial Disclosure: The authors declared that this study received no financial support.

\section{References}

1. Monheit GD. Consultation for photo-aging skin. 2001;19:401-403.

2. Cavalié M, Sillard L, Montaudié H, Bahadoran P, Lacour JP, Passeron T. Treatment of keloids with laser-assisted topical steroid delivery: a retrospective study of 23 cases. Dermatol Ther 2015;28:74-78.

3. Balci DD, Inandi T, Dogramaci CA, Celik E. DLQI scores in patients with keloids and hypertrophic scars: a prospective case control study. J Dtsch Dermato Ges 2009;7:688-692.

4. Mahdavian Delavary B, van der Veer WM, Ferreira JA, Niessen FB. Formation of hypertrophic scars: evolution and susceptibility. J Plast Surg Hand Surg 2012;46:95-101.

5. Wolfram D, Tzankov A, Pülzl P, Piza-Katzer H. Hypertrophic scars and keloids--a review of their pathophysiology, risk factors, and therapeutic management. Dermatol Surg 2009;35:171-181.

6. Arno Al, Gauglitz GG, Barret JP, Jeschke MG. Up-to-date approach to manage keloids and hypertrophic scars: a useful guide. Burns 2014;40:1255-1266. 
7. Slemp AE, Kirschner RE. Keloids and scars: a review of keloids and scars, their pathogenesis, risk factors, and management. Curr Opin Pediatr 2006;18:396402.

8. Leventhal D, Furr M, Reiter D. Treatment of keloids and hypertrophic scars: a meta-analysis and review of the literature. Arch Facial Plast Surg 2006;8:362368.

9. Tawfic SO, El-Tawdy A, Shalaby S, Foad A, Shaker O, Sayed SS, Metwally D. Evaluation of Fractional CO2 Versus Long Pulsed Nd:YAG Lasers in Treatment of Hypertrophic Scars and Keloids: A Randomized Clinical Trial. Lasers Surg Med 2020;52:959-965.
10. Koike S, Akaishi S, Nagashima Y, Dohi T, Hyakusoku H, Ogawa R. Nd:YAG Laser Treatment for Keloids and Hypertrophic Scars: An Analysis of 102 Cases. Plast Reconstr Surg Glob Open 2015;2:e272.

11. Al-Mohamady Ael-S, Ibrahim SM, Muhammad MM. Pulsed dye laser versus long-pulsed Nd:YAG laser in the treatment of hypertrophic scars and keloid: A comparative randomized split-scar trial. J Cosmet Laser Ther 2016;18:208212.

12. Choi JE, Oh GN, Kim JY, Seo SH, Ahn HH, Kye YC. Ablative fractional laser treatment for hypertrophic scars: comparison between Er:YAG and $\mathrm{CO} 2$ fractional lasers. J Dermatolog Treat 2014;25:299-303. 